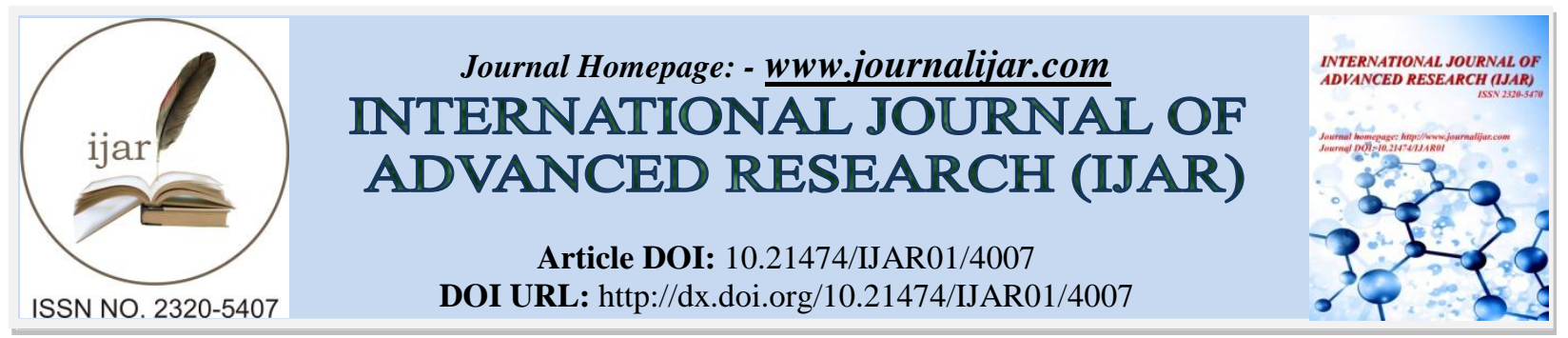

RESEARCH ARTICLE

\title{
REVIEW ON CFD ANALYSIS OF DOUBLE TUBE HELICAL COIL HEAT EXCHANGER.
}

\section{Vikas Singh $^{1}$, Subhashini Verma ${ }^{1}$, Priyanshu Dradhomar ${ }^{1}$, Pallavi Dradhomar ${ }^{1}$ and Manjunatha. $\mathbf{M}^{\mathbf{2}}$.}

1. Department of Mechanical Engineering, JSS Academy of Technical Education, Noida, India.

2. Assistant Professor, Department of Mechanical Engineering, JSS Academy of Technical Education, Noida, India.

\section{Manuscript Info}

Manuscript History

Received: 15 February 2017

Final Accepted: 13 March 2017

Published: April 2017

\begin{abstract}
This literature review focuses on the study and applications of Computational Fluid Dynamics (CFD) in the field of heat exchangers. It has been observed cfd analysis is a very useful tool in the field of heat exchangers to determine the heat transfer characteristics. Different models available in general purpose commercial CFD tools i.e. standard, realizable, energy model, radiation and k- $\varepsilon$ (2eqn.), with velocity-pressure coupling schemes such as SIMPLEC have been adopted to carry out the simulations. The qualities of the solutions obtained from these simulations arewithin the acceptable range with very accurate results. Hence proving CFD as an effective tool for predicting the behavior and performance of a wide variety of heat exchangers.
\end{abstract}

Copy Right, IJAR, 2017,. All rights reserved.

\section{Introduction:-}

Heat exchangers play an important role in the operation of systems such as process industries, power plants and heat recovery units. Its inevitable need has necessitated work on efficient and reliable designs leading towards optimum share in the overall system performance. The Log Mean Temperature Difference (LMTD) and the number of heat transfer units (NTU) method are two methods used for design of heat exchanger. These methods have some limitations i.e. iterative in nature and need of a prototype to implement the design. Due to these reasons, these methods are time consuming as well as uneconomical especially for large scale models. However, economical access to powerful microprocessors has created a way for the evaluation of Computational Fluid Dynamics (CFD). CFD is a science that can help to study fluid flow, heat transfer, chemical reactions etc. by solving mathematical equations using numerical analysis. It is also very helpful in designing a heat exchanger system from scratch and in troubleshooting or optimization of the design. CFD employs a very simple principle of resolving the entire system into small cells or grids and applying governing equations on these discrete elements to find numerical solutions regarding pressure distribution, temperature gradients and flow parameters in a shorter time and at a lower cost, as the required experimental work is reduced.

The results obtained with the CFD are of acceptable quality. In the current work, various parameters changes have been done in the design of heat exchangers and their solutions with the help of CFD have been reviewed. This work can serve as a ready reference for application of CFD in design of various types of heat exchangers.

Corresponding Author:- Vikas Singh.

Address:- Department of Mechanical Engineering, JSS Academy of Technical Education, Noida, India. 


\section{Types of heat exchangers:-}

There are innumerable types of heat exchangers in use. It is not limited to plate type heat exchanger, shell-and-tube heat exchanger, etc. but broad classification of heat exchangers based on their construction is outlined in the Fig. 1 below. The double tube helical coil heat exchanger is given in fig.2. The heat exchanger in the figure is a 2 turn heat exchanger.

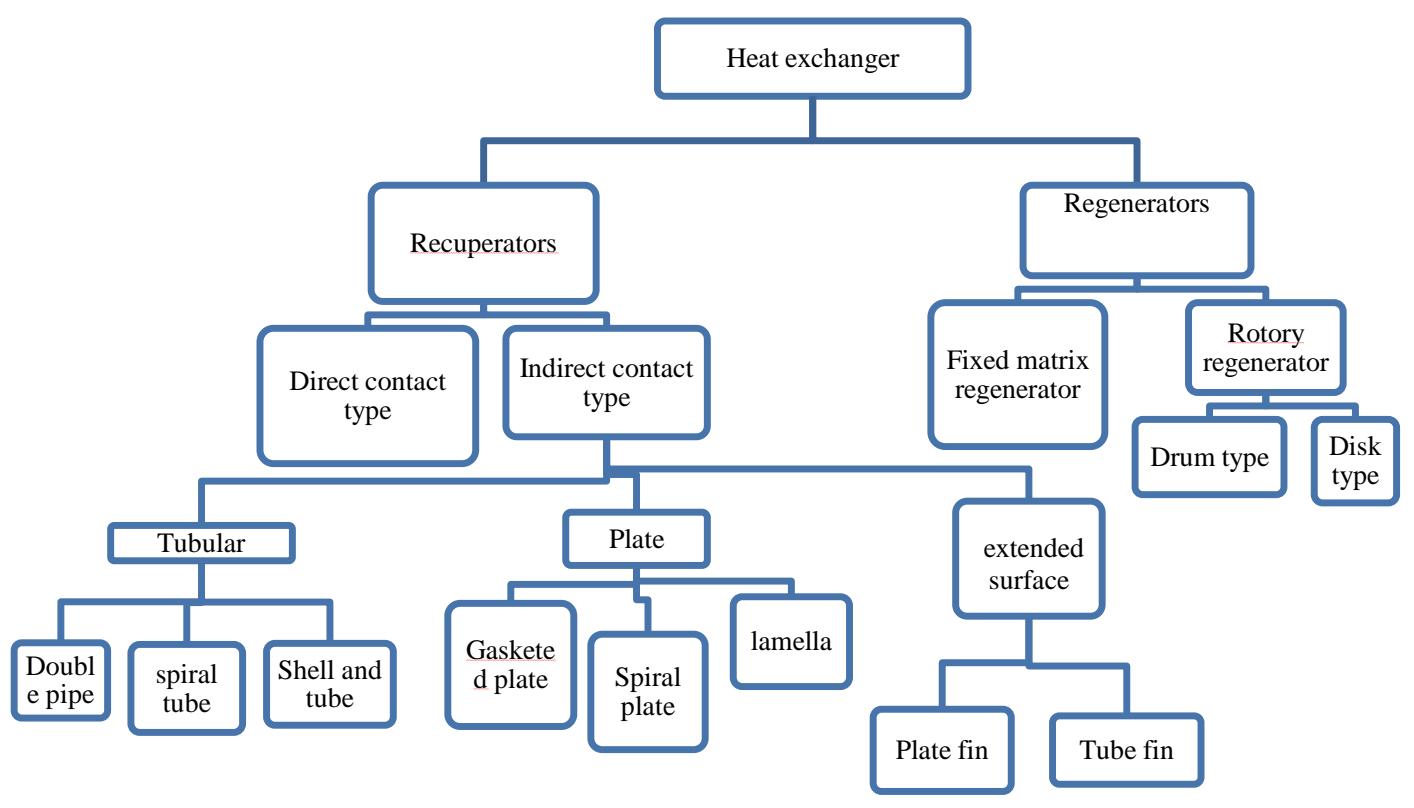

\section{ANSYS \\ R15.0}

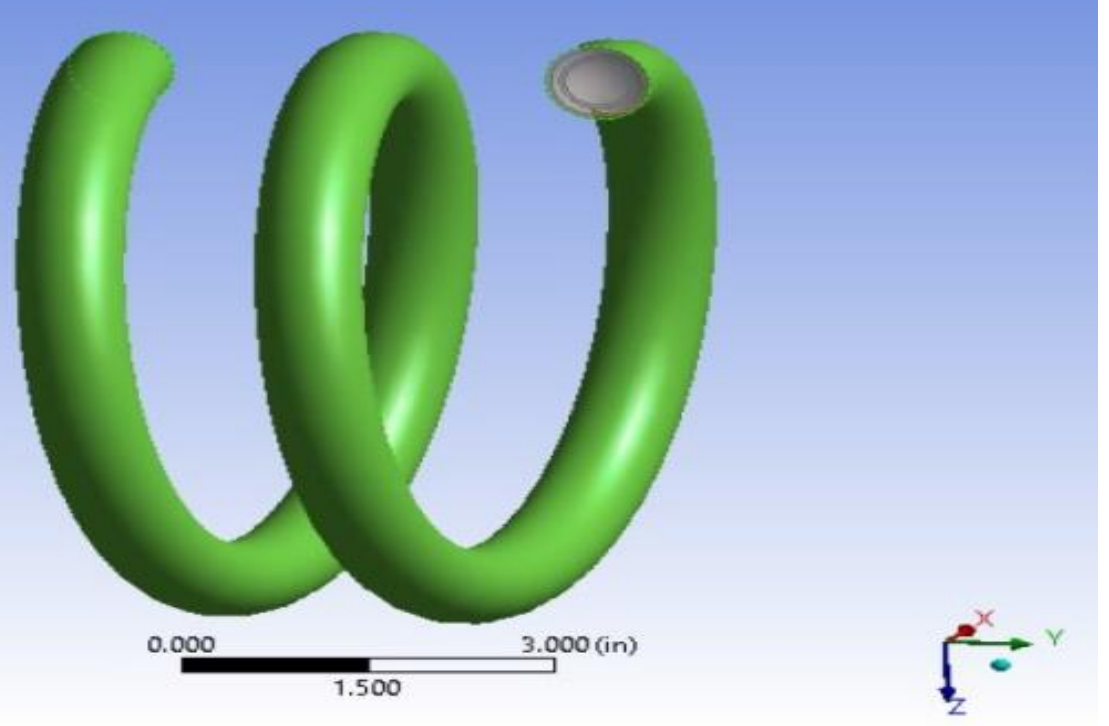




\section{Literature Review:-}

D. G. Prabhanjanet al. found that use of a helical coil heat exchanger result in increase of heat transfer coefficient compared to a similarly dimensioned straight tube heat exchanger. They also observed that both heat exchangers had higher heat transfer coefficients when the bath temperature was increased, may be due to increased buoyancy effects. Flaw rate did not affect the heat transfer coefficient; due to the fact that the flow was turbulent and increasing the flow rate does not greatly change the wall effects. Temperature rise of the fluid was found to be effected by coil geometry and by the flow rate.

J.S. Jayakumara, et al. observed the following Characteristics of heat transfer under turbulent flow of singlephasewater through helical coil heat exchanger.

Analysis was carried out for both the constant wall temperature and constant wall heat flux boundary conditions. Fluid particles were found to undergo oscillatory motion inside the pipe and this caused fluctuations in heat transfer rate.

Nusselt number on the outer side of the coil i.e. far from coil axis wasfound to be highest among all other points at a specified cross-section, while that at the inner side of the coil is the lowest.

A number of numerical experiments were carried out to study influence of coil parameters, viz., pitch circle diameter, coil pitch and pipe diameteronheat transfer characteristics. The coil pitch was found to behaving significance only in the developing section of heat transfer.

The torsional forces induced by the pitch causes oscillations in the Nusselt number. However, the average Nusselt number wasnot affected by the coil pitch. Hence correlation for estimation of Nusselt number for applications to helical coils shall include the pitch circle radius and pipe diameter apart from the flow parameters and physical properties.

Vimal Kumar et al. performedexperimental as well as numerical study for coil-in-coil heat exchanger on the pilot plant scale. Theyvaried both mass flow rate in the inner tube as well as in the annulus and the counter flow configuration was tested. It was observed that the overall heat transfer coefficient increases with increase in the inner-coiled tube Dean number for a constant flow rate in the annulus region. Similar trends in the variation of overall heat transfer coefficient were observed for different flow rates in the annulus region for a constant flow rate in inner-coiled tube.The numerical predictions for hydrodynamics and fully developed heat transfer were found to be verifying experimental results. The friction factor value in the inner-coiled tube was verified with the numerical work and literature data. The annulus side friction factor values were alsofound to be within close tolerence with the numerical predictions.

Nasser Ghorbani et al. found through an experimental investigation of mixed convection heat transfer in helically coiled tube heat exchanger as the most applicable compact heat exchangers. They obtainedresults indicating ifRm is greater than unity then the temperature profiles will be of quadratic form from bottom to top of the heat exchanger. The profiles were found to be linear for Rmclose to unity. when the mass flow rate ratio was very less than unity, then temperature profiles were of the logarithmic form. The $\varepsilon^{-N T U}$ relation of the mixed convection heat exchangers was found to be of a pure counter-flow heat exchanger.

J.S. Jayakumara,et al.observed through their studies that the use of constant values for the thermaland transport properties of the heat transport mediumresults in prediction of inaccurate heat transfer coefficients. They also predicted that heat transfer in a situation of fluid-to fluidheat transfer in a heat exchanger does not requires arbitrary boundary conditions such as constant wall temperature,constant heat flux, etc. henceit is required to model the equipment consideringconjugate heat transfer. An experimental setup was fabricatedto study fluid-fluid heat transfer in a helically coiled heatexchanger. Heat transfer characteristics of the heat exchangerwith helical coil was studied using the CFD code FLUENT.The CFD predictions matchedwell with theexperimental results within error limits. Basedon the results a correlation was developed to calculate theinner heat transfer coefficient of the helical coil.

Rahul Kharat et al. proposed that a modified equation can be used to analyze the effect of different designing parameters on heat transfer coefficient. Among them two most important design parameters are number of turns and tube diameter for the study of their effect on heat transfer performance. They observed in their studies that the heat 
transfer coefficient decreases with the decrease in number of turns. To analyze the effect of tube diameter on the heat transfer coefficients, the heat transfer coefficients were evaluated for the different tube diameter keeping inner coil pitch circle diameter, outer coil pitch circle diameter and velocity constant. These results shows that the heat transfer coefficient increases with the increase in tube diameter. This result is quite interesting and does not agree with the expected flow behaviour. This is primarily due to reduction in coil gap with increasing tube diameter and the effect of tube diameter is not dissociated with the effect of coil gap. The effect of coil gap was found to be dominating over the effect of the tube diameter.

J.S. Jayakumaraet al.validatedheat transfer calculations for the two-phase flow against experimental results of flow through an annular pipe. Studies were carried out by varying (i) coil pitch, (ii) pipe diameter (iii) pitch circle diameter and (iv) void fraction at the inlet. Unlike the flow through a straight pipe, the centrifugal force caused due to the curvature of the pipe causes heavier fluid (water phase) to flow along the outer side of the pipe. Hence high velocity and high temperature were observed along the outer side. They observed that torsion caused by pitch of the coil madethe flow unsymmetrical about the horizontal plane of coil. As the pitch was increased, higher velocity and higher temperature regions were observed on the bottom half of the pipe. They also found that increase in pipe diameter causes higher heat transfer coefficient and lower pressure dropkeeping the inlet velocity constant. This effect is due to the influence of secondary flows. As the pitch circle diameter was increased, the centrifugal forces decreases and this causes reduction of heat transfer coefficient anddrop in pressure.

Vikas Kumar et al.considered a cross flow air to air tube type heat exchanger and observed the relation between heat transfer and Dean's Number keeping the flow rate constant in the outer region. An increase in heat transfer from was observed with a rise in inner De. A assumption was made that heat lost occurred only through convection from the top surface of the exchanger.whereas all radiation losses were neglected in the study.

Carla S. Fernandes et al, presented different correlations for $\mathrm{Nu}$, Re and Pr and discussed the fluid entry effect on average $\mathrm{Nu}$ for stirred yogurt during cooling in a PHE. They employed HerscheleBulkley model and numerical analysis was carried out by first considering and then ignoring the temperature effect on the viscosity. A comparative analysis with past literature made them to conclude that $\mathrm{Nu}$ experiences an increase with an increase in the Re.

\section{Conclusions:-}

1. By reviewing different journals it was observed that the double tube helical coil heat exchanger has greater heat transfer rate as compared to straight double tube heat exchanger.

2. It requires less space for same temperature dropi.e. it is compact. This is highly useful as we can use in plants which have less area.

3. Due to centrifugal action high velocity and high temperature is observed outside.

4. CFD is found to be very useful tool for the analysis of double tube helical coil heat exchanger as results obtained from CFD analysis were withinclose tolerance with practical values.

5. It was observed that epsilon 2-eqn model gives most accurate result for this heat exchanger.

\section{References:-}

1. D. G. Prabhanjan, G. S. V. Ragbavan and T. J. Kennic,.2002. Comparisionof heat transfer rates between a straight tube heat exchanger and a helically coiled heat exchanger. International Comm. Heat transfer,volume. 29. No.2. pp. 185-191.

2. J.S. Jayakumara, S.M. Mahajania, J.C. Mandala, Kannan N. Iyera, P.K. Vijayan,.2010. CFD analysis of singlephase flows inside helically coiled tubes. Computers and Chemical Engineering 34. 430-446.

3. Vimal Kumar, SupreetSaini, Manish Sharma, K.D.P. Nigam.,2006. Pressure drop and heat transfer study in tube-in-tube helical heat exchanger. Chemical Engineering Science. 4403 - 4416.

4. Nasser Ghorbani ,HessamTaherian, MofidGorji, HessamMirgolbabaei ,.2010. An experimental study of thermal performance of shell-and-coil heat exchangers. International Communications in Heat and Mass Transfer 37. 775-781.

5. J.S. Jayakumar, S.M. Mahajani, J.C. Mandal, P.K. Vijayan, Rohidas Bhoi,.2008. Experimental and CFD estimation of heat transfer in helically coiled heat exchangers.chemical engineering research and design 86. 221-232. 
6. Rahul Kharat, NitinBhardwaj, R.S. Jha,.2009. Development of heat transfer coefficient correlation for concentrichelical coil heat exchanger. International Journal of Thermal Sciences 48. 2300-2308.

7. J.S.Jayakumar, S.M.Mahajani, J.C. Mandal, Kannan N. Iyer, P.K. Vijayan,.2010.Thermal hydraulic characteristics of air-water two -phaseflows in helical pipes.chemical engineering research and design Vol.88.501-512.

8. V. Kumar, D. Gangacharyulu, P.M. Rao, R.S. Barve,.2003 CFD analysis of cross flow air to air tube type heat exchanger.<http://www.cham.co.uk/PUC/PUC_Melbourne/papers/Paper18_Kumar.pdf>(accessed 23.04.11.).

9. C.S. Fernandes, R.P. Dias, J.M. Nobrega, I.M. Afonso, L.F. Melo, J.M. Maia,.2006. Thermal behavior of stirred yoghurt during cooling in plate heat exchangers, J. Food Eng. 76 .433-439. 